\section{Adaptação transcultural e validação do Questionário de Atitudes Socioculturais em Relação à Aparência-4 (SATAQ-4) aplicado a estudantes universitários}

\author{
Cross-cultural adaptation of the Sociocultural \\ Attitudes Towards Appearance Scale-4 (SATAQ-4) \\ applied to university students
}

\author{
Adaptación transcultural y validación del Cuestionario \\ de Actitudes Socioculturales en Relación con \\ la Apariencia-4 (SATAQ-4) aplicado a \\ estudiantes universitarios
}

QUESTÕES METODOLÓGICAS

METHODOLOGICAL ISSUES
Júlia Valério Barra 1

Wanderson Roberto da Silva 1

João Marôco 2

Juliana Alvares Duarte Bonini Campos 1

doi: $10.1590 / 0102-311 \times 00170218$

\title{
Resumo
}

O objetivo deste estudo foi traduzir e adaptar culturalmente o Questionário de Atitudes Socioculturais em Relação à Aparência-4 (SATAQ-4) para a lingua portuguesa e avaliar sua validade e confiabilidade quando aplicado a estudantes brasileiros. Realizou-se a validação de face, de conteúdo e pré-teste. Para a avaliação do indice de incompreensão, participaram do pré-teste 91 estudantes de três países de língua portuguesa (25 brasileiros, 37 portugueses e 29 moçambicanos). A validade de construto foi estimada utilizando-se estratégia confirmatória com os índices razão de qui-quadrado pelos graus de liberdade $\left(\chi^{2} / g l\right)$, comparative fit index (CFI), Tucker-Lewis index (TLI) $e$ root mean square error of approximation (RMSEA). A confiabilidade foi estimada. A invariância foi estimada para amostras independentes, para o sexo e presença/ausência de sobrepeso/obesidade. Todos os itens do SATAQ-4 foram compreendidos pelos estudantes do pré-teste. No estudo principal, participaram 1.051 universitários brasileiros (idade $=20,9$ anos; $D P=2$, 4; $66,4 \%$ mulheres). O SATAQ-4 apresentou adequada validade $\left(\chi^{2} / g l=8,39\right.$; $C F I=0,98 ; T L I=0,98 ; R M S E A=0,08)$ e confiabilidade (alfa de Cronbach = $0,84-0,95)$ na amostra. O modelo apresentou invariância entre amostras independentes e foi não invariante segundo sexo e presença/ausência de sobrepeso/obesidade. Conclui-se que o SATAQ-4é um instrumento válido e confiável para amostra de universitários brasileiros, sendo uma alternativa para a avaliação das atitudes socioculturais em relação à aparência nesta população.

Imagem Corporal; Características Culturais; Psicometria;

Estudos de Validação; Questionários

\author{
Correspondência \\ J. A. D. B. Campos \\ Departamento de Alimentos e Nutrição, Faculdade de Ciências \\ Farmacêuticas de Araraquara, Universidade Estadual Paulista \\ Júlio de Mesquita Filho. \\ Rod. Araraquara-Jaú, Km 01, s/n, Campos Ville Araraquara, SP \\ 14800-903, Brasil. \\ jucampos@fcfar.unesp.br \\ 1 Faculdade de Ciências Farmacêuticas de Araraquara,
Universidade Estadual Paulista Júlio de Mesquita Filho,
Araraquara, Brasil.
2 Instituto Superior de Psicologia Aplicada, Lisboa, Portugal.
}




\section{Introdução}

A imagem corporal é um conceito complexo definido por Schilder ${ }^{1}$ (p. 26) como "a figuração do nosso corpo formada em nossa mente". Aspectos fisiológicos, biológicos, psicológicos e sociológicos estão envolvidos na estruturação desse conceito 2,3. Entre esses, pode-se destacar a relevância do aspecto sociológico, que diz respeito à influência das relações sociais e da mídia na formação da imagem corporal 3 e tem sido fortemente associado à insatisfação com a aparência 4 . Essa insatisfação pode se relacionar ao desenvolvimento de distúrbios alimentares e dismórficos corporais 5 , práticas não saudáveis para o controle de peso 2,6, baixa autoestima, dificuldades interpessoais e ansiedade social 7. Alguns estudos estimam que $25 \%$ a $80 \%$ dos indivíduos estão insatisfeitos com a aparência física 4,8 , sendo a população de jovens e adolescentes a de maior risco para o desenvolvimento desses transtornos 6 .

O modelo mais utilizado para explicar a influência das pressões socioculturais na insatisfação corporal é o Modelo Tripartite proposto por Thompson et al. 9 em 1999. Esse modelo sugere que os indivíduos são pressionados a alcançar ideais de aparência culturalmente adotados 6,9 principalmente por três influências sociais (família, amigos e mídia). Esse fato pode fazer com que os indivíduos internalizem um ideal de aparência socialmente imposto ${ }^{5}$. A internalização refere-se ao quanto um indivíduo aceita os ideais de aparência e expressa o desejo de alcançá-los, passando a ter pensamentos e comportamentos que visem a atender a este ideal, na maior parte das vezes, inatingíveis 10 .

Estudos da literatura 6,10 ressaltam que a internalização dos padrões diferencia de acordo com o sexo, sendo a magreza o padrão ideal para mulheres e o corpo musculoso para os homens. Contudo, deve-se enfatizar que os ideais de aparência vão além do desejo de ter um corpo magro ou atlético. Os indivíduos buscam atender a um padrão em relação à forma do corpo, sendo relevante, dentro deste padrão corporal, aspectos como a quantidade de gordura corporal e a distribuição da gordura no corpo 11 .

Para avaliar a influência das pressões socioculturais para que se atinja um padrão corporal ideal e a internalização deste ideal, podem ser utilizados instrumentos psicométricos a fim de identificar o risco de desenvolvimento de transtornos ligados à imagem corporal. Entre esses instrumentos podese citar a Tripartite Influence Scale (TIS), composta por 39 itens capazes de avaliar a influência sociocultural da mídia, amigos e família na imagem corporal 12 e o Sociocultural Attitudes Towards Appearance Questionnaire-4(SATAQ-4), composto por 22 itens capazes de avaliar a internalização do corpo magro e muscular/atlético e a influência da mídia, amigos e família na imagem corporal 6. Esse último instrumento torna-se interessante por ser capaz de avaliar as pressões e a internalização simultaneamente.

A primeira versão desse instrumento foi elaborada em 1995 por Heiberg et al. 13 e revisada em 1997 por Cusomano \& Thompson ${ }^{14}$. Essas versões continham apenas itens relacionados à internalização dos ideais de aparência e a conscientização da influência sociocultural na formação da imagem corporal 13,14. Posteriormente, com o objetivo de englobar outros aspectos relacionados à influência sociocultural na imagem corporal, o SATAQ foi revisado por Thompson et al. 15. Essa nova versão foi intitulada SATAQ-3 e incluiu itens sobre internalização atlética, pressão da mídia e informação, sendo composta por quatro fatores ("internalização geral", "internalização atlética", "pressão" e "informação") 15 e amplamente utilizada em diferentes países 15,16,17,18,19,20, aplicada também em amostras da população brasileira 21,22,23,24.

A fim de melhorar algumas limitações conceituais do instrumento, Schaefer et al. 6 realizaram a revisão do SATAQ-3 e propuseram uma nova versão, denominada SATAQ-4. Nessa versão, foram incluídos itens sobre a pressão da família e amigos, uma vez que estas são influências sociais importantes na internalização do padrão corporal ${ }^{6}$. Além disso, houve a inclusão de itens que tratam especificamente da internalização do corpo magro/baixa gordura corporal e internalização do corpo muscular/atlético, com o objetivo de abranger também a população masculina, que possui maior preocupação com a muscularidade.

Posteriormente, outra versão do instrumento foi proposta por Schaefer et al. 25, o SATAQ-4R. Nessa versão, incluiu-se mais um fator de pressão sociocultural, referente a pares românticos, vizinhos e professores. Foram retirados os itens dos fatores de internalização que remetiam a comportamentos, restando apenas itens referentes ao aspecto cognitivo. Também foram retirados os itens contendo a palavra "atlético" devido à possível ambiguidade do termo. O SATAQ-4R tem duas versões distintas, sendo uma voltada para a população masculina e outra para a feminina. 
Para o presente estudo, o SATAQ-4 foi selecionado como instrumento de medida por ser capaz de identificar a internalização e as pressões socioculturais em relação à aparência tanto em homens quanto em mulheres, usando a mesma versão do instrumento, facilitando, portanto, sua aplicação em amostras alargadas. Além disso, essa versão foi amplamente utilizada em amostras de homens e mulheres de vários países, apresentando adequada validade e confiabilidade 6,26,27,28 e, até o momento, ainda não há versão em português desse instrumento. Outra vantagem apontada na literatura do SATAQ-4 é a existência de ponto de corte para o fator "Internalização - Magro/Pouca gordura corporal", o que auxilia na identificação de risco de desenvolvimento de transtornos alimentares/dismórficos corporais nos indivíduos, contribuindo para a intervenção e tratamento clínico dos mesmos 10 .

Dessa forma, realizou-se este estudo com o objetivo de elaborar uma versão em português do SATAQ-4, adaptando-o culturalmente para a utilização em diferentes contextos da língua portuguesa e avaliar sua validade e confiabilidade quando aplicado à amostra de estudantes universitários brasileiros.

\section{Métodos}

Para alcançar os objetivos do estudo, a metodologia foi dividida em duas etapas. A primeira trata da adaptação transcultural do SATAQ-4 e foi constituída pela tradução, retrotradução, validade de face e conteúdo do instrumento e pré-teste. A segunda etapa constou da avaliação da validade e confiabilidade da versão adaptada culturalmente do SATAQ-4 aplicada a estudantes universitários. Essas etapas estão descritas a seguir.

\section{Variáveis de estudo e instrumento de medida}

Para a caracterização da amostra foram levantadas informações como sexo, idade, área e ano do curso (Humanas/Sociais, Exatas, Saúde/Biológicas), nível econômico, peso e altura. O nível econômico dos participantes foi estimado utilizando-se o Critério de Classificação Econômica Brasil (Associação Brasileira de Empresas de Pesquisa - ABEP; http://www.abep.org/criterio-brasil). O peso e a altura foram relatados pelos participantes e, posteriormente, estimado o índice de massa corporal (IMC) para a classificação do estado nutricional 29.

As atitudes socioculturais em relação à aparência foram estimadas usando-se como instrumento de medida o SATAQ-4 que foi elaborado, originalmente, na língua inglesa por Schaefer et al. 6. O instrumento apresenta 22 itens com respostas em escala de Likert de 5 pontos (variando de 1- discordo totalmente a 5- concordo totalmente). Os itens são distribuídos em 5 fatores ("Internalização - Magro/Pouca gordura corporal" - itens: 3, 4, 5, 8, 9; "Internalização - Muscular/Atlético" - itens: 1, 2, 6, 7, 10; "Pressão - Família" - itens: 11, 12, 13, 14; "Pressão - Colegas" - itens: 15, 16, 17, 18; "Pressão - Mídia” - itens: 19, 20, 21, 22).

\section{Etapa 1: tradução e adaptação cultural}

\section{- Tradução, retrotradução e validade de face}

Inicialmente, foi solicitada permissão ao autor original do SATAQ-4 para a tradução e adaptação cultural do instrumento e esta foi concedida por escrito. O processo de tradução do SATAQ-4 foi realizado seguindo os preceitos metodológicos sugeridos por Beaton et al. ${ }^{30}$. Quatro tradutores nativos da língua portuguesa (2 brasileiros e 2 portugueses) com conhecimento do contexto cultural do país de origem do instrumento e que atuam nas áreas de imagem corporal, psicometria e saúde realizaram a tradução do instrumento de maneira independente. As traduções foram comparadas pelos autores do presente estudo, buscando elaborar uma única versão em português do instrumento, que foi denominada "versão intermediária". Essa versão foi elaborada seguindo o acordo ortográfico estabelecido entre os países de língua portuguesa em 2009. A versão intermediária foi submetida à retrotradução. A retrotradução foi realizada por nativo na língua inglesa, com conhecimento do português e atuante na área de psicologia e saúde. Cabe esclarecer que o retrotradutor não tinha conhecimento da 
versão original do instrumento e que o mesmo não foi informado de que se tratava de um processo de retrotradução. A versão retrotraduzida foi comparada à versão original em inglês do SATAQ-4 por 1 especialista em psicometria, 1 especialista em imagem corporal e 1 especialista em língua portuguesa, para verificar sua equivalência.

\section{- Índice de validade de conteúdo}

Após essa etapa, a versão em português obtida foi apresentada a 4 especialistas (das áreas de Saúde/ Psicologia), sendo 3 brasileiros e 1 português, para a avaliação da equivalência (semântica, idiomática, cultural e conceitual) 30 de cada item do SATAQ-4 frente ao instrumento original (em inglês). A avaliação foi realizada individualmente e de forma independente.

Os especialistas assinalaram em uma escala do tipo Likert de 4 pontos (1: "sentença não equivalente"; 2: "impossível avaliar a equivalência da sentença sem que esta seja revista"; 3: "sentença equivalente, mas que necessita de alterações menores"; 4: "sentença totalmente equivalente") suas opiniões em relação às equivalências 31 .

Baseando-se nas respostas obtidas, foi calculado o Índice de Validade de Conteúdo (IVC). Esse índice considera a proporção de juízes que assinalaram as respostas 3 e 4 , e se IVC $\geq 80 \%$ é atestada a equivalência. Para aqueles itens com IVC $<80 \%$ foram realizadas alterações com base nos comentários dos especialistas ${ }^{31}$. Depois de realizadas as alterações pertinentes, a versão do instrumento foi considerada finalizada para a realização do pré-teste.

\section{- Pré-teste}

A versão em português conciliada do SATAQ-4 foi pré-testada em grupos de estudantes universitários de três diferentes países de língua portuguesa (Brasil, Portugal e Moçambique) para estimar o tempo de preenchimento e o Índice de Incompreensão (II) da versão culturalmente adaptada. Tal índice é realizado para verificar, na população-alvo, as dúvidas e a compreensão relativas aos itens do instrumento 32 .

A amostra do pré-teste foi composta por 91 estudantes universitários dos diferentes países, sendo $70,33 \%$ do sexo feminino e média de idades de 21,70 (DP = 4,48) anos. Dos participantes, 25 eram brasileiros da Universidade Estadual Paulista Júlio de Mesquita Filho (Unesp), campus de Araraquara (64\% sexo feminino; idade: 20,9 (DP = 3,3) anos); 37 portugueses da Escola Superior de Comunicação Social de Lisboa ( $72 \%$ sexo feminino; idade de 19,51 ( $\mathrm{DP}=1,70$ ) anos) e 29 moçambicanos da Universidade Pedagógica de Moçambique (72\% sexo feminino; idade: 25,37 (DP = 5,26) anos).

A versão pré-teste do SATAQ-4 foi autopreenchida pelos estudantes. Foi informado pelo pesquisador principal deste estudo e por escrito que se tratava de uma avaliação da compreensão de cada item. Caso o participante não compreendesse algum item/palavra ou o sentido/linguagem usado foi solicitado ao mesmo que sua dúvida fosse inserida por escrito no espaço intitulado "Sugestão", para que as mesmas pudessem ser avaliadas pelos autores do presente trabalho. Frente à obtenção de II $\leq$ $20 \%$ para todos os itens, a versão em português do SATAQ- 4 foi considerada finalizada 33.

\section{Etapa 2: avaliação das características psicométricas}

\section{- Desenho de estudo e delineamento amostral}

Trata-se de estudo metodológico, com delineamento amostral não probabilístico por conveniência. Foram convidados a participar estudantes matriculados nos cursos de graduação da Faculdade de Ciências Farmacêuticas e da Faculdade de Ciências e Letras da Unesp (campus de Araraquara). Foram incluídos no trabalho indivíduos de 18 a 30 anos de idade, que preencheram corretamente e assinaram o Termo de Consentimento Livre e Esclarecido. Aqueles que não preencheram na íntegra o SATAQ-4 e que não informaram idade, peso e/ou altura foram excluídos.

O cálculo do tamanho mínimo da amostra para a realização das análises estatísticas foi realizado com base na proposta de Hair Jr. et al. 34, que recomendam a utilização de 5 a 10 sujeitos por parâmetro do modelo a ser estimado. No presente trabalho, foi utilizado o SATAQ-4, composto por 
54 parâmetros (22 itens, 22 erros e 10 correlações entre os fatores). Assim, o tamanho mínimo da amostra estimado foi de 270 a 540 sujeitos. Considerando uma taxa de perda de $20 \%$, o tamanho amostral mínimo foi corrigido para 338 a 676 sujeitos.

Dos estudantes convidados, 1.159 concordaram em participar do estudo. Porém, 108 (9,32\%) foram excluídos da amostra por não preencherem corretamente o SATAQ-4, não informarem idade, peso e/ou altura ou terem mais de 30 anos de idade.

As propriedades psicométricas do SATAQ-4 aplicado à amostra foram avaliadas baseando-se na estimativa das validades de construto e confiabilidade, descritas a seguir.

\section{- Validade de construto}

A validade de construto do SATAQ-4 foi avaliada com base nas validades fatorial, convergente e discriminante, com o intuito de verificar a adequação do modelo teórico proposto para SATAQ-4 à amostra.

A validade fatorial foi estimada por meio de análise fatorial confirmatória (AFC) com o método de estimação dos mínimos quadrados ponderados ajustado para média e variância (WLSMV), implementado no programa MPLUS 7.11 (https://www.statmodel.com/). Como índices para avaliação da qualidade de ajustamento foram usados a razão de qui-quadrado pelos graus de liberdade ( $\left.\chi^{2} / \mathrm{gl}\right)$, o comparative fit index (CFI), Tucker-Lewis index (TLI) e o root mean square error of approximation (RMSEA). $\mathrm{O}$ ajustamento do modelo aos dados foi considerado adequado quando os pesos fatoriais $(\lambda) \geq 0,40$, $\chi^{2} / \mathrm{gl} \leq 2,0$, CFI e TLI $\geq 0,90$ e RMSEA $\leq 0,1035$. A validade convergente foi estimada baseando-se na variância extraída média (VEM), seguindo a proposta de Fornell \& Larcker 36 e considerada adequada se $V E M \geq 0,50$. A validade discriminante foi avaliada por análise correlacional e foi considerado indicativo de adequada validade discriminante quando VEMi e VEMj forem maiores do que o quadrado da correlação entre estes fatores 36 .

\section{- Invariância fatorial}

Após ajustamento do modelo fatorial aos dados, foi estimada a invariância deste modelo em amostras independentes para verificar sua validade externa. Para tanto, a amostra total foi subdividida de forma aleatória em duas partes iguais, denominadas “Teste" $(n=526)$ e "Validação" $(n=525)$. Para estimar a invariância métrica, escalar e estrita foi realizada análise multigrupos com a estatística teste da diferença de qui-quadrados $\left(\Delta \chi^{2}\right) 35$. Considerou-se a invariância métrica (fraca) quando os pesos fatoriais não diferiram significativamente entre as amostras $\left(\Delta \chi^{2} \lambda\right.$ : p $\left.>0,05\right)$, invariância escalar (forte) quando os pesos fatoriais e os limiares não diferiram significativamente $\left(\Delta \chi^{2} \lambda\right.$ e $\left.\Delta \chi^{2} \mathrm{i}: \mathrm{p}>0,05\right)$ e invariância estrita (muito forte) quando pesos fatoriais, os limiares e as variâncias/covariâncias dos resíduos não diferiram significativamente $\left(\Delta \chi^{2} \lambda, \Delta \chi^{2}\right.$ i e $\left.\Delta \chi^{2} \operatorname{cov}: \mathrm{p}>0,05\right) 35$.

Também foi analisada a invariância do SATAQ-4 entre grupos, segundo sexo, dividindo a amostra entre homens $(n=353)$ e mulheres $(n=698)$ e segundo estado nutricional, sendo a amostra subdividida em "ausência de sobrepeso/obesidade - IMC $<25 \mathrm{~kg} / \mathrm{m}^{2}$ " $(\mathrm{n}=758)$ e "presença de sobrepeso/ obesidade - IMC $\geq 25 \mathrm{~kg} / \mathrm{m}^{2}$ " ( $\left.\mathrm{n}=293\right)$. Foi realizada análise multigrupos com a estatística teste da diferença de qui-quadrado $\left(\Delta \chi^{2}\right)$, conforme descrito antes 35 .

\section{Confiabilidade}

A confiabilidade foi avaliada baseando-se na consistência interna e na confiabilidade composta (CC). A consistência interna foi estimada usando-se o Coeficiente alfa ordinal $(\alpha)$ calculado na matriz de correlação policórica 37, utilizando-se o programa RStudio 3.4.3 (https://www.rstudio.com/pro ducts/rstudio/download/). A confiabilidade composta foi estimada seguindo a proposta de Fornell \& Larcker 36. Foi considerado indicativo de adequada confiabilidade se $\alpha$ e CC $\geq 0,70$. 


\section{Procedimentos e aspetos éticos}

O SATAQ-4 e o questionário sociodemográfico foram autopreenchidos pelos estudantes em horários habituais de aula, e foi solicitada autorização dos professores para a realização do estudo. Participaram os estudantes que concordaram voluntariamente em colaborar e que assinaram o Termo de Consentimento Livre e Esclarecido. Os pesquisadores esclareceram que a participação era facultativa e anônima. A realização deste trabalho foi aprovada pelo Comitê de Ética em Pesquisa em Seres Humanos da Faculdade de Ciências Farmacêuticas - Unesp (CAAE: 63553516.4.0000.5426).

\section{Resultados}

A versão em português culturalmente adaptada do SATAQ-4 está na Tabela 1.

Cabe esclarecer que no processo de tradução/retrotradução o advérbio "definitely" encontrado na versão original (definitely desagree/agree) foi traduzido para o português como "totalmente", para melhor entendimento pela população-alvo. Os adjetivos "thin" e "lean" que estão nas questões 3, 5 e 8 receberam a mesma tradução no português por serem sinônimos, sendo descritos como "magro(a)". A expressão "encourage me" encontrada nos itens 13,14 e 15, que normalmente é utilizada no Brasil como "me encorajam", foi traduzida como "incentivam/encorajam-me" para a adaptação transcultural de Portugal e Moçambique. Além disso, a expressão "the Media”, referente aos meios de comunicação, foi descrita de maneiras diferentes entre os países. No Brasil, deve-se utilizar a expressão "a mídia”, já em Portugal e Moçambique esta deve ser substituída por "os média”, para que haja melhor compreensão dos itens.

Todos os itens foram considerados adequados pelos especialistas (IVC $\geq 80 \%$ ), sendo, portanto, a versão do instrumento considerada finalizada para a realização do pré-teste.

O tempo médio de preenchimento do SATAQ-4 no pré-teste $(\mathrm{n}=91)$ foi de 6,16 $(\mathrm{DP}=3,50)$ minutos (brasileiros: 4,55 (DP = 2,14), portugueses: 5,21( $\mathrm{DP}=2,47)$, moçambicanos: 8,85 $(\mathrm{DP}=4,10)$. Os estudantes não apresentaram dúvidas referentes à compreensão dos itens, obtendo-se assim II $\leq 20 \%$. Dessa maneira, a versão em português conciliada do instrumento foi considerada finalizada e apta para utilização.

Depois de estabelecida a versão em português adaptada culturalmente, esta foi aplicada aos estudantes brasileiros participantes do estudo $(\mathrm{n}=1.051)$.

A média de idades dos estudantes foi de 20,93 anos (DP = 2,38) e o IMC médio de 23,29kg/ $\mathrm{m}^{2}$ $(\mathrm{DP}=4,03)$. A caracterização demográfica da amostra está descrita na Tabela 2. Cabe esclarecer que alguns participantes não relataram todas as informações demográficas e, portanto, a análise descritiva não contém a amostra total em todas as variáveis.

Observa-se que os estudantes são majoritariamente do sexo feminino, da área de Ciências Humanas, possuem peso normal para altura (eutrofia) e encontram-se na classe econômica B.

Na Figura 1, apresenta-se o modelo fatorial do SATAQ-4 ajustado à amostra total ( $\mathrm{n}=1.051$; $\lambda=0,729-0,976 ; \chi^{2} / \mathrm{gl}=8,397 ; \mathrm{CFI}=0,980 ; \mathrm{TLI}=0,977 ; \mathrm{RMSEA}=0,084 ;$ IC90\%: 0,080-0,088; $\mathrm{VEM}=0,622-0,921 ; \mathrm{r}=0,063-0,590 ; \mathrm{CC}=0,912-0,977 ; \alpha=0,897-0,975$ ).

$\mathrm{O}$ ajustamento do modelo aos dados foi considerado adequado, assim como os pesos fatoriais $(\lambda)$ dos itens, a validade convergente, a validade discriminante e a confiabilidade do instrumento.

Quando realizada a análise multigrupos em amostras independentes (Teste x Validação), o instrumento apresentou invariância fatorial estrita $\left(\Delta \chi^{2}: \mathrm{p} \lambda=0,138 ; \mathrm{pi}=0,218\right.$ e pcov $\left.=0,286\right)$. Com relação à invariância entre grupos, esta foi rejeitada $(\mathrm{p}<0,001)$ tanto entre os sexos quanto entre presença/ausência de sobrepeso/obesidade. Na Tabela 3, apresenta-se o ajustamento do SATAQ-4 às subamostras.

Pode-se notar adequado ajustamento do SATAQ-4 às subamostras. Vale ressaltar que apesar dos valores de $\chi^{2} / \mathrm{gl}$ serem exacerbados, tal fato ocorre pelo tamanho amostral alargado, e não deve ser considerado de forma isolada para aceitar ou rejeitar o ajustamento do modelo 35. 
Tabela 1

Versão original e versão em português culturalmente adaptada do Questionário de Atitudes Socioculturais em Relação à Aparência (SATAQ-4).

\begin{tabular}{|c|c|}
\hline Item & Versão original em inglês * \\
\hline & $\begin{array}{l}\text { Directions: please read each of the following items carefully and indicate } \\
\text { the number that best reflects your agreement with the statement. }\end{array}$ \\
\hline & Definitely disagree $=1$ \\
\hline & Mostly disagree $=2$ \\
\hline & Neither agree nor disagree $=3$ \\
\hline & Mostly agree $=4$ \\
\hline & Definitely agree $=5$ \\
\hline 1 & It is important for me to look athletic. \\
\hline 2 & I think a lot about looking muscular. \\
\hline 3 & I want my body to look very thin. \\
\hline 4 & I want my body to look like it has little fat. \\
\hline 5 & I think a lot about looking thin. \\
\hline 6 & I spend a lot of time doing things to look more athletic. \\
\hline 7 & I think a lot about looking athletic. \\
\hline 8 & I want my body to look very lean. \\
\hline 9 & I think a lot about having very little body fat. \\
\hline 10 & I spend a lot of time doing things to look more muscular. \\
\hline
\end{tabular}

Answer the following questions with relevance to your Family (include: parents, brothers, sisters, relatives):

I feel pressure from family members to improve my appearance.

Family members encourage me to decrease my level of body fat.

Family members encourage me to get in better shape.

Answer the following questions with relevance to your Peers (include: close friends, classmates, other social contacts):

My peers encourage me to get thinner.

I feel pressure from my peers to improve my appearance.

I feel pressure from my peers to look in better shape. I get pressure from my peers to decrease my level of body fat.

Answer the following questions with relevance to the media (include: television, magazines, the Internet, movies, billboards, and advertisements):

I feel pressure from the media to look in better shape.

I feel pressure from the media to look thinner.

I feel pressure from the media to improve my appearance.

I feel pressure from the media to decrease my level of body fat.
Versão em português

Instruções: Por favor, leia cuidadosamente cada um dos

itens e assinale o número que melhor identifique sua concordância com a sentença.

Discordo totalmente $=1$

Discordo em parte $=2$

Não concordo nem discordo $=3$

Concordo em parte $=4$

Concordo totalmente $=5$

É importante para mim ter um porte atlético.

Eu penso muito em ter um porte musculoso.

Eu quero que o meu corpo aparente ser muito magro.

Eu quero que o meu corpo aparente ter pouca gordura.

Eu penso muito em ter uma aparência magra.

Eu passo muito tempo fazendo coisas para ter uma aparência mais atlética.

Eu penso muito em ter uma aparência atlética.

Eu quero que o meu corpo tenha uma aparência muito magra.

Eu penso muito sobre ter muito pouca gordura corporal.

Eu passo muito tempo fazendo coisas para aparentar ter um porte mais musculoso.

Responda às perguntas a seguir considerando a sua família (incluir: pais, irmãos, irmãs, parentes):

Eu sinto pressão de pessoas da minha família para parecer mais magro(a).

Eu sinto pressão de pessoas da minha família para melhorar minha aparência.

As pessoas da minha família encorajam/estimulam-me a diminuir meu nível de gordura corporal.

Algumas pessoas da minha família encorajam-me a ter uma forma física melhor.

Responda às perguntas a seguir considerando seus colegas (incluir: amigos próximos, colegas de turma, outros contatos sociais):

Meus colegas encorajam-me a ficar mais magro(a).

Eu sinto pressão dos meus colegas para melhorar a minha aparência.

Eu sinto pressão dos meus colegas para ter uma forma melhor. Eu sinto pressão dos meus colegas para diminuir meu nível de gordura corporal.

Responda às perguntas a seguir considerando a mídia (os média) ** (incluir: televisão, revistas, internet, filmes, outdoors e anúncios/propagandas):

Eu sinto pressão da mídia (dos média) ** para ter uma forma melhor.

Eu sinto pressão da mídia (dos média) ** para parecer mais magro.

Eu sinto pressão da mídia (dos média) ** para melhorar minha aparência.

Eu sinto pressão da mídia (dos média) ** para diminuir meu nível de gordura corporal.

* Schaefer et al. 6.

** Em Portugal e Moçambique, deve-se substituir a expressão "da mídia" pela expressão entre parênteses "dos média". 
Tabela 2

Caracterização demográfica dos estudantes universitários participantes do estudo.

\begin{tabular}{lc}
\hline Características & $\mathbf{n}(\%)$ \\
\hline Sexo & \\
Masculino & $353(33,6)$ \\
Feminino & $698(66,4)$ \\
Área do curso & \\
$\quad$ Biologia/Saúde & $243(23,1)$ \\
Exatas & $95(9,0)$ \\
Humanas & $713(67,9)$ \\
Ano do curso & \\
10 & $300(28,8)$ \\
20 & $304(29,1)$ \\
3o & $206(19,7)$ \\
4o e 5o & $234(22,4)$ \\
Estado nutricional & \\
Baixo peso & $82(7,8)$ \\
Eutrofia & $676(64,3)$ \\
Sobrepeso & $220(20,9)$ \\
Obesidade & $73(7,0)$ \\
Estrato econômico (renda média familiar estimada) * & \\
A (R\$ 23.345,11) & \\
B (R\$ 7.874,85) & $264(25,1)$ \\
C (R $\$$ 2.328,56) & $600(57,1)$ \\
D-E (R\$ 708,00) & $181(17,2)$ \\
\hline
\end{tabular}

* Critério de Classificação Econômica Brasil; 2018 (Associação Brasileira de Empresas de Pesquisa - ABEP; http://www. abep.org/criterio-brasil).

\section{Discussão}

A versão traduzida e adaptada culturalmente para o português conciliado do SATAQ-4 apresentada neste estudo permitiu que o instrumento fosse aplicado e validado para a amostra de estudantes universitários brasileiros. Com base nessa versão, abre-se espaço para que o instrumento possa ser aplicado, também, em amostras de outros países de língua portuguesa, possibilitando comparações culturais em contextos mais alargados.

Os resultados de validade e confiabilidade adequados encontrados no presente trabalho estão em consonância com os resultados encontrados por Schaefer et al. 6 no estudo de validação original do SATAQ-4 e com os demais estudos realizados com estudantes universitários em diferentes países 26,27,28. O modelo teórico de cinco fatores 6,26,27,28 foi testado e se mostrou adequado também neste trabalho, tanto para a amostra total quanto para as subamostras testadas. A invariância observada entre amostras independentes apontou para adequada validade externa deste modelo fatorial. Esses resultados sugerem que o SATAQ-4 mede, de forma semelhante, a internalização dos ideais de aparência e as pressões socioculturais em relação à aparência quando utilizada em amostras advindas da mesma população.

Com relação às subamostras, observou-se que a invariância foi refutada tanto paro o sexo quanto para presença/ausência de sobrepeso/obesidade. Isso indica que o SATAQ-4 operacionaliza os conceitos de maneira diferente de acordo com o grupo avaliado. Consequentemente, o padrão corporal e as pressões socioculturais em relação à aparência têm diferentes relevâncias para as mulheres e para os homens e para indivíduos com presença/ausência de sobrepeso/obesidade, inviabilizando, portanto, uma comparação direta dos escores obtidos com a SATAQ-4 entre os diferentes grupos 36 . 
Figura 1

Estrutura fatorial do modelo do Questionário de Atitudes Socioculturais em Relação à Aparência-4 (SATAQ-4) em amostra de estudantes universitários brasileiros.

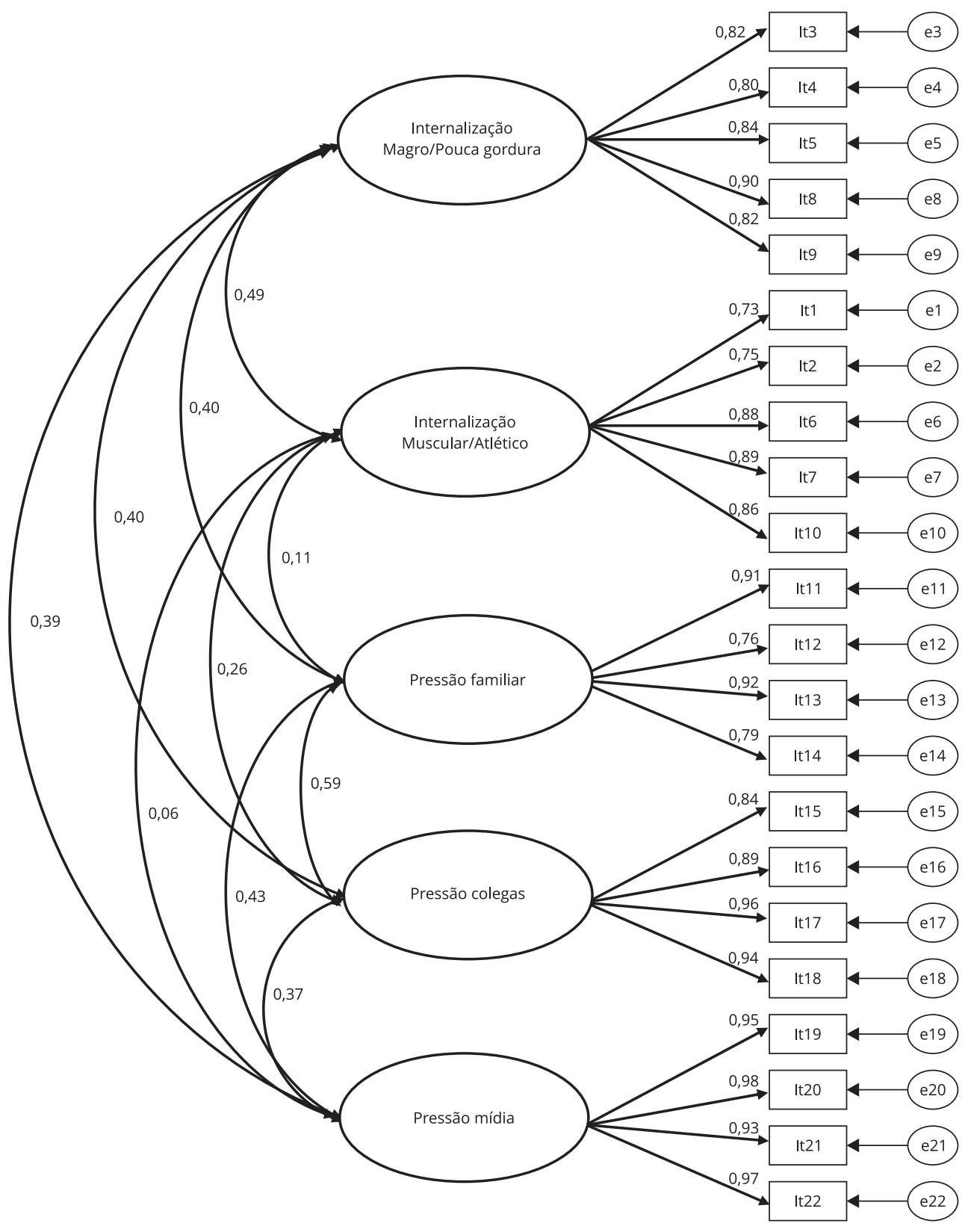

Esse resultado corrobora com os dados encontrados na literatura, em que é apontado que a maneira com que são sentidas as pressões socioculturais e o padrão ideal de aparência é diferente para homens e mulheres 6,10, o que implica a necessidade de utilização de instrumentos, como o SATAQ-4, que consigam capturar de forma distinta os conceitos, segundo o sexo do indivíduo. Do mesmo modo, a variabilidade observada entre as amostras com presença/ausência de sobrepeso/obesidade era 
Tabela 3

Indicadores para avaliação das propriedades psicométricas do Questionário Sociocultural em Relação à Aparência-4 (SATAQ-4) ajustado às amostras Teste, Validação, Masculina, Feminina, Ausência de Sobrepeso/Obesidade e Presença de Sobrepeso/Obesidade.

\begin{tabular}{|c|c|c|c|c|c|c|c|c|c|c|}
\hline Subamostra & $\mathbf{n}$ & $\lambda$ & $\chi^{2} / g \mid$ & CFI & TLI & RMSEA (IC90\%) & $r$ & VEM & $\mathrm{CC}$ & $\mathbf{a}$ \\
\hline Teste & 525 & $0,72-0,98$ & 4,779 & 0,984 & 0,982 & $0,085(0,080-0,090)$ & $0,00-0,61$ & $0,67-0,93$ & $0,91-0,98$ & $0,89-0,98$ \\
\hline Validação & 526 & $0,73-0,97$ & 4,475 & 0,977 & 0,974 & $0,081(0,076-0,087)$ & $0,13-0,56$ & $0,68-0,90$ & $0,91-0,97$ & $0,90-0,97$ \\
\hline Masculino & 353 & $0,69-0,97$ & 4,039 & 0,978 & 0,975 & $0,093(0,086-0,010)$ & $0,04-0,61$ & $0,62-0,92$ & $0,88-0,98$ & $0,86-0,98$ \\
\hline Feminino & 698 & $0,68-0,98$ & 5,104 & 0,981 & 0,978 & $0,077(0,072-0,081)$ & $0,21-0,61$ & $0,72-0,90$ & $0,90-0,97$ & $0,89-0,97$ \\
\hline \multicolumn{11}{|l|}{ Sobrepeso/ } \\
\hline \multicolumn{11}{|l|}{ Obesidade } \\
\hline Ausente & 758 & $0,67-0,97$ & 6,285 & 0,976 & 0,972 & $0,083(0,079-0,088)$ & $0,11-0,57$ & $0,65-0,91$ & $0,87-0,97$ & $0,87-0,97$ \\
\hline Presente & 293 & $0,73-1,00$ & 2,999 & 0,993 & 0,992 & $0,083(0,075-0,090)$ & $-0,03-0,50$ & $0,65-0,96$ & $0,90-0,99$ & $0,89-0,99$ \\
\hline
\end{tabular}

a: coeficiente alfa ordinal; $\lambda$ : pesos fatoriais; $\chi^{2} / g l$ : qui-quadrado pelos graus de liberdade; CC: confiabilidade composta; CFI: comparative fit index; IC90\%: intervalo de 90\% de confiança; r: correlação entre os fatores; RMSEA: root mean square error of approximation; TLI: Tucker-Lewis index; VEM: variância extraída média.

esperada, pois, segundo a literatura 38, indivíduos com sobrepeso e obesidade possuem maior preocupação com a aparência e são mais insatisfeitos com o corpo do que indivíduos eutróficos, sendo mais propensos a internalizar um padrão de corpo magro. Portanto, o SATAQ-4 foi capaz de operacionalizar de maneira diferente também por estado nutricional, o que é de suma importância para identificar o risco de desenvolvimento de transtornos alimentares e dismórficos corporais nos diferentes grupos e a contribuição das atitudes socioculturais em relação à aparência. Desse modo, o SATAQ-4 pode ser utilizado para o levantamento de informações acuradas que podem ser relevantes para a elaboração de programas de prevenção e tratamento de distúrbios alimentares e dismórficos corporais específicos para cada grupo.

O estudo tem algumas limitações, como o fato de a escolha da instituição para a composição da amostra não ter sido probabilística, o que pode dificultar a generalização dos resultados. Outra limitação foi a restrição da idade dos participantes, que impede uma comparação entre os conceitos analisados para jovens e adultos mais velhos. Assim, sugerimos que trabalhos futuros busquem comparar indivíduos de diferentes idades, culturas e etnias.

Apesar das limitações, o estudo apresenta, pela primeira vez, a versão desse instrumento na língua portuguesa, viabilizando a utilização do SATAQ-4 em jovens brasileiros, e se mostrando válido e confiável para tal amostra. Além disso, essa versão foi elaborada em consonância com outros dois países de língua portuguesa, com diferentes características sociais, culturais e econômicas, o que permite sua aplicação em outros contextos, possibilitando comparações culturais e a realização de estudos transnacionais, ampliando, assim, as discussões acerca da importância da internalização do corpo ideal e das pressões socioculturais em relação à aparência.

Dessa maneira, o SATAQ-4 pode ser uma alternativa para o rastreamento de risco para o desenvolvimento de transtornos alimentares e dismórficos corporais, com base na avaliação da internalização de padrões corporais ideais devido às pressões socioculturais. Tal fato torna-se relevante para profissionais e pesquisadores da área, no sentido de ampliar a reflexão e construção de estratégias de manejo de diagnóstico e/ou tratamento mais direcionadas.

\section{Conclusão}

A versão em português do SATAQ-4 apresentou validade e confiabilidade adequadas para a amostra de estudantes universitários brasileiros, sendo distinta a avaliação das atitudes socioculturais em relação à aparência de acordo com o sexo e a presença/ausência de sobrepeso/obesidade. 


\section{Colaboradores}

J. V. Barra contribui na concepção do projeto, coleta dos dados, análise e interpretação dos dados, redação do artigo e revisão crítica do conteúdo intelectual, aprovação da versão final a ser publicada. W. R. Silva e J. Marôco contribuíram na análise e interpretação dos dados, redação do artigo e revisão crítica do conteúdo intelectual, aprovação da versão final a ser publicada. J. A. D. B. Campos contribuiu na concepção do projeto, análise e interpretação dos dados, redação do artigo e revisão crítica do conteúdo intelectual, aprovação da versão final a ser publicada.

\section{Informações adicionais}

ORCID: Júlia Valério Barra (0000-0002-85539691); Wanderson Roberto da Silva (0000-00018897-8772); João Marôco (0000-0001-9214-5378); Juliana Alvares Duarte Bonini Campos (0000-00017123-5585)

\section{Agradecimentos}

À Fundação de Amparo à Pesquisa do Estado de São Paulo - Fapesp (Grant \#2017/18679-0) e ao Conselho Nacional de Desenvolvimento Científico e Tecnológico - CNPq (Grant\#131073/2017-6) pelo financiamento do estudo. Ao Professor Doutor Benvindo Felismino Samuel Maloa, da Universidade Pedagógica de Moçambique. Departamento de Psicologia, Colégio de Ciências Educacionais pelos dados coletados em Moçambique para a realização do pré-teste.

\section{Referências}

1. Schilder P. A imagem do corpo: as energias construtivas da psique. São Paulo: Editora Martins Fontes; 1994.

2. Cash TF, Smolak L. Body image: handbook of science, practice, and prevention. 2nd Ed. Nova York: The Guilford Press; 2012.

3. Ferreira MEC, Castro MR, Morgado FFR. Imagem corporal: reflexões, diretrizes e práticas de pesquisa. Juiz de Fora: Editora UFJF; 2014.

4. Silva WR, Dias JCR, Pimenta F, Campos JADB. Avaliação da preocupação com a forma corporal: um desafio. Psychology, Community \& Health 2014; 3:103-19.

5. Cafri G, Yamamiya Y, Brannick M, Thompson JK. The influence of sociocultural factors on body image: a meta-analysis. Clinical Psychology: Science and Practice 2005; 12:421-33.

6. Schaefer LM, Burke NL, Thompson JK, Dedrick RF, Heinberg LJ, Calogero RM, et al. Development and validation of the Sociocultural Attitudes Towards Appearance Questionnaire-4 (SATAQ-4). Psychol Assess 2015; 27:54-67.

7. Dakanalis A, Zanetti AM, Riva G, Colmegna F, Volpato C, Madeddu F, et al. Male body dissatisfaction and eating disorder symptomatology: moderating variables among men. J Health Psychol 2015; 20:80-90.

8. Striegel-Moore RH, Rosselli F, Perrin N, De Bar L, Wilson GT, May A, et al. Gender difference in the prevalence of eating disorder symptoms. Int J Eat Disord 2009; 42:471-4.

9. Thompson JK, Heinberg LJ, Altabe M, Tantleff-Dunn S. Exacting beauty: Theory, assessment, and treatment of body image disturbance. Washington DC: American Psychological Association; 1999.

10. Schaefer LM, Burke NL, Thompson JK. Thinideal internalization: how much is too much? Eat Weight Disord 2018; [Epub ahead of print].

11. Santos LAS. O corpo, o comer e a comida: um estudo sobre as práticas corporais alimentares cotidianas a partir da cidade de Salvador Bahia. Salvador: EDUFBA; 2008.

12. Keery H, van den Berg P, Thompson JK. An evaluation of the tripartite influence model of body dissatisfaction and eating disturbance with adolescent girls. Body Image 2004; 1:23751.

13. Heinberg LJ, Thompson JK, Stormer S. Development and validation of the Sociocultural Attitudes Towards Appearance Questionnaire. Int J Eat Disord 1995; 17:81-9.

14. Cusumano DL, Thompson JK. Body image and body shape ideals in magazines: Exposure, awareness, and internalization. Sex Roles 1997; 37:701-21.

15. Thompson JK, van den Berg P, Roehrig M, Guarda AS, Heinberg LJ. The sociocultural attitudes towards appearance scale-3 (SATAQ-3): development and validation. Int J Eat Disord 2004; 35:293-304. 
16. Stefanile C, Matera C, Nerini A, Pisani E. Validation of an Italian version of the Sociocultural Attitudes Towards Appearance Questionnaire-3 (SATAQ-3) on adolescent girls. Body Image 2011; 8:432-6.

17. Swami V. An examination of the factor structure of the Sociocultural Attitudes Towards Appearance Questionnaire-3 in Malaysia. Body Image 2009; 6:129-32.

18. Rousseau A, Valls M, Chabrol H. Validation of the French version of the Sociocultural Attitudes Towards Appearance Scale-3 (SATAQ-3). Encephale 2010; 36:270-6.

19. Llorente E, Warren CS, de Eulate LP, Gleaves DH. A Spanish version of the Sociocultural Attitudes Towards Appearance Questionnaire-3 (SATAQ-3): translation and psychometric evaluation. J Clin Psychol 2013; 69:240-51.

20. Argyrides M, Kkeli N, Kendeou P. Validation of the factor structure of the Greek adaptation of the Sociocultural Attitudes Towards Appearance Questionnaire (SATAQ-3). Body Image 2014; 11:201-5.

21. Amaral ACS, Cordas TA, Conti MA, Ferreira MEC. Equivalência semântica e avaliação da consistência interna da versão em português do Sociocultural Attitudes Towards Appearance Questionnaire-3 (SATAQ-3). Cad Saúde Pública 2011; 27:1487-97.

22. Amaral AC, Ribeiro MS, Conti MA, Ferreira CS, Ferreira ME. Psychometric evaluation of the Sociocultural Attitudes Towards Appearance Questionnaire-3 among Brazilian young adults. Span J Psychol 2013; 16:E94.

23. Amaral ACS, Ferreira MEC. Body dissatisfaction and associated factors among Brazilian adolescents: a longitudinal study. Body Image 2017; 22:32-8.

24. Fortes LS, Ferreira ME, Oliveira SM, Cyrino ES, Almeida SS. A socio-sports model of disordered eating among Brazilian male athletes. Appetite 2015; 92:29-35.

25. Schaefer LM, Harriger JA, Heinberg LJ, Soderberg T, Kevin Thompson J. Development and validation of the sociocultural attitudes towards appearance questionnaire-4-revised (SATAQ-4R). Int J Eat Disord 2017; 50:104-17.

26. Llorente E, Gleaves DH, Warren CS, Perezde-Eulate L, Rakhkovskaya L. Translation and validation of a Spanish version of the Sociocultural Attitudes Towards Appearance Questionnaire-4 (SATAQ-4). Int J Eat Disord 2015; 48:170-5.
27. Rodgers RF, Schaefer LM, Thompson JK, Girard M, Bertrand M, Chabrol H. Psychometric properties of the Sociocultural Attitudes Towards Appearance Questionnaire-4 (SATAQ-4) in French women and men. Body Image 2016; 17:143-51.

28. Yamamiya Y, Shimai S, Schaefer LM, Thompson JK, Shroff H, Sharma R, et al. Psychometric properties and validation of the Sociocultural Attitudes Towards Appearance Questionnaire-4 (SATAQ-4) with a sample of Japanese adolescent girls. Body Image 2016; 19:89-97.

29. World Health Organization. Obesity: preventing and managing the global epidemic. Geneva: World Health Organization; 2000. (WHO Technical Report Series, 894).

30. Beaton D, Bombardier C, Guillemin F, Ferraz $\mathrm{MB}$. Recommendations for the cross-cultural adaptation of the DASH and QuickDASH outcome measures. Toronto: Institute for Work \& Health; 2007.

31. Alexandre NM, Coluci MZ. Content validity in the development and adaptation processes of measurement instruments. Ciênc Saúde Colet $2011 ; 16: 3061-8$.

32. Nusbaum L, Natour J, Ferraz MB, Goldenberg J. Translation, adaptation and validation of the Roland-Morris questionnaire - Brazil RolandMorris. Braz J Med Biol Res 2001; 34:203-10.

33. Spexoto MC, Serrano SV, Halliday V, Maroco J, Campos JA. Cancer Appetite and Symptom Questionnaire (CASQ) for Brazilian patients: cross-cultural adaptation and validation study. PLoS One 2016; 11:e0156288.

34. Hair Jr. JF, Black WC, Babin B, Anderson RE, Tatham RL. Multivariate data analysis. $6^{\text {th }}$ Ed. New Jersey: Prentice Hall; 2005.

35. Maroco J. Análise de equações estruturais: fundamentos teóricos, software e aplicações. 2a Ed. Pêro Pinheiro: ReportNumber; 2014.

36. Fornell C, Larcker DF. Evaluating SEM with unobserved variables and measurment error. Journal of Marketing Research 1981; 18:39-50.

37. Gadermann AM, Guhn M, Zumbo BD. Estimating ordinal reliability for Likert-type and ordinal item response data: a conceptual, empirical, and practical guide. Practical Assessment, Research \& Evaluation 2012; 17:1-13.

38. Cecon RS, Franceschini S, Peluzio M, Hermsdorff HHM, Priore SE. Overweight and body image perception in adolescents with triage of eating disorders. ScientificWorldJournal 2017; 2017:8257329. 


\section{Abstract}

The aim of this study was to translate and culturally adapt the Sociocultural Attitudes Towards Appearance Scale-4 (SATAQ-4) to the Portuguese language and assess its validity and reliability when applied to Brazilian students. Face, content, and pretest validity were assessed. In order to assess the levels of misunderstanding, a pretest was performed with 91 students from three Portuguese-speaking countries (25 Brazilians, 37 Portuguese, and 29 Mozambicans). Construct validity was estimated using a confirmatory strategy with chi-square by degrees of freedom ratio $\left(\chi^{2} / d f\right)$, comparative fit index (CFI), Tucker-Lewis index (TLI), and root mean square error of approximation (RMSEA). The reliability was estimated. Invariance was estimated for independent samples, for sex and presence/absence of overweight/obesity. Students participating in the pretest understood all the items in SATAQ-4. Participation in the main study included 1,051 Brazilian university students (age $=20.9$ years $(S D=2.4), 66.4 \%$ women). SATAQ-4 presented adequate validity $\left(\chi^{2} / d f=8.39 ; C F I=0.98, T L I=0.98, R M S E A\right.$ $=0.08)$ and reliability (Cronbach's alpha $=0.84-$ $0.95)$ in the sample. The model showed invariance between independent samples and was not invariant according to sex or presence/absence of overweight/obesity. In conclusion, SATAQ-4 is a valid and reliable instrument for a sample of Brazilian university students, as an alternative for assessing sociocultural attitudes towards appearance in this population.

Body Image; Cultural Characteristics; Psychometrics; Validations Studies;

Questionnaires

\section{Resumen}

El objetivo de este estudio fue traducir a la lengua portuguesa y adaptar culturalmente el Cuestionario de Actitudes Socioculturales en Relación con la Apariencia-4 (SATAQ-4), y evaluar su validez y confiabilidad cuando se aplica a estudiantes brasileños. Se realizó la validación del diseño, contenido y pre-examen. Para la evaluación del indice de incomprensión, participaron en el pre-examen 91 estudiantes de tres países de lengua portuguesa ( 25 brasileños, 37 portugueses y $29 \mathrm{mo}$ zambiqueños). La validez de constructo se estimó utilizando la estrategia confirmatoria con los indices de razón chi-cuadrado por los grados de libertad $\left(\chi^{2} / g l\right)$, el comparative fit index (CFI), TuckerLewis index (TLI) $y$ root mean square error of approximation (RMSEA). Asimismo, se estimó la confiabilidad. La invarianza se estimó para muestras independientes, para el sexo y presencia/ ausencia de sobrepeso/obesidad. Los estudiantes del pre-examen comprendieron todos los items del SATAQ-4. En el estudio principal, participaron 1.051 universitarios brasileños (edad $=20,9$; $D P=2,4$; años, 66, 4\% mujeres). El SATAQ-4 presentó una adecuada validez $\left(\chi^{2} / g l=8,39\right.$; $C F I=0,98 ; T L I=0,98 ; R M S E A=0,08)$ y confiabilidad (alfa de Cronbach $=0,84-0,95$ ) en la muestra. El modelo presentó invarianza entre muestras independientes y fue no invariante según sexo $y$ presencia/ausencia de sobrepeso/obesidad. Se concluye que el SATAQ-4 es un instrumento válido y confiable para la muestra de universitarios brasileños, siendo una alternativa para la evaluación de las actitudes socioculturales, en relación con la apariencia en esta población.

Imagen Corporal; Características Culturais; Psicometría; Estudios de Validación; Cuestionários
Recebido em 29/Ago/2018

Versão final reapresentada em 01/Fev/2019

Aprovado em 28/Fev/2019 